\title{
Articles
}

Central European Review of Economics \& Finance

Vol. 17, No. 1(2017), pp. 53-63, D0I: 10.24136/ceref.2017.003

Ivan Dimitrov ${ }^{1}$, Adile Dimitrova ${ }^{2}$, Petko Yangyozov $^{3}$

\section{APPROACH OF ASSESSMENT THE PRIORITY OF SUPPLY LOGISTICS SUB-PROCESSES IMPROVEMENT}

The aim of this research paper is to present an approach of assessment the priorities for improvements of each supply logistics sub-process in the organization. The ways of comparison of targets sub-processes and their corresponding real sub-processes are explained. Algorithm for calculating of priority of supply logistics sub-process improvement is reviewed. It includes calculating of vector that describes the necessity of sub-process improvement in each measurement as well as determining of aggregate coefficient that represents the need for each supply logistics sub-process optimization in all its measurements.

JEL Classification Codes: L23.

Keywords: ssupply logistics, sub-process, optimization, methodology, priority of improvement, algorithm.

\section{Introduction}

The function of each enterprise is to carry out transformation of inputs (raw materials and supplies), through the production factors (buildings, machines, labor), into a product/service designated to satisfy the customer's need (Angelov, 2008). Transformation is related to the running of various business processes (Harmon, 2007), processes (Deckler, 2003; Haist, 2001;

\footnotetext{
${ }^{1}$ Prof PhD. in „Prof. Dr. Assen Zlatarov” University - Burgas.

${ }^{2}$ Assistant PhD. in „Prof. Dr. Assen Zlatarov” University - Burgas.

${ }^{3}$ Assistant PhD. in „Prof. Dr. Assen Zlatarov” University - Burgas.
} 
Harrington, 1991; Ould, 2006; Lowenthal, 2003) and activities (McDonald, 2010; Portougal, 2006), united in production cycles. Logistics processes are an important part of business processes in the organization divided into three phases - logistics of supply, logistics of production and logistics of distribution (Dimitrov, 2004). In this connection, they can be viewed as specific business processes across the organization. Each phase of the logistics can be further decomposed into several levels in case of expanding of the analysis. Logistics processes crossing through individual units and they are oriented along to the information and materials flow passing through the supply chain (Rosemann, 2006). For improvement of logistics activities in the organization mathematical apparatus that allows the identification and reorganization of the critical elements in logistics processes can be used. The feedback which established customer's satisfaction by logistics service and the amount of logistics costs, provides the necessary signal that starts process of logistics system improving. This signal moves in the opposite direction of the running logistics processes. In order to respond the customer's needs, organizations starts optimization in one or all logistics phases. The optimization should be carried out with the help of methodology in conformity with the company structure, as well as with the strategy chosen.

In order to implement optimization of the supply logistics in the organization, it is necessary to determine whether actual need of improvement exists. The signal is broadcast from the production system of the organization which is supply logistics customer within the overall logistics process.

One way to establish guidelines for improvement is by applying the approach of defining of general necessity of supply logistics improvement. To that end, it is necessary the actual and the desired state of the processes of supply logistics to be presented by vectors - real and target ones. The real vector represents an aggregate of elements, describes all processes and activities building the supply logistics (Brüggemann, 1998). Each element is represented as a partial vector with the relevant coordinates. The coordinates describe the real values of the parameters characterizing various aspects of process effectiveness (Papula, 2001). By summing up the vectors the common (resultant) vector is obtained. The target vector has been built by marking the coordinates of the goal on the coordinate system, the measurements of which are defined by the parameters derived from feedback received from customers. From the initial point of the coordinate system to the point marking the desired improvement a vector is built, called target vector. If comparison between the vector which represent the real process and the vector which represent the target process shows deviation in favor 
of the target vector, then it is necessary to perform a thoroughgoing analysis and improvement of the supply logistics process. Otherwise, it is assumed that the parameters of the existing company process of supply logistics are better than the goal set forth; therefore, improvement is not needed. The comparison between coordinates of both vectors enables the determination of the overall necessity of improvement as well the necessity of sub-process improvement for supply logistics' process. Furthermore, it can be assessment the necessity of improvement of each sub-process, which builds up the entire supply logistics process through calculating their efficiency beside the target goal. In order to achieve overall and sustainable improvements, it is necessary optimization to pass sequential the following steps:

- assessment of the overall necessity of supply logistics improvement;

- assessment of the necessity of sub-process improvement;

- assessment of the priority of sub-process improvement.

The aim of the paper is to present an approach of assessment the priority of supply logistics' sub-process improvement in the organization.

\section{Identification of the priority of supply logistics sub-process improvement}

The identification of priority of the existing supply logistics sub-processes improvement is done by comparison between the partial real and target vectors. Various parameters characterizing the efficiency of the supply logistics may be selected as measurements, such as "accuracy of deliveries from suppliers”, „time of supply”, "high maintenance”, „low process's costs”, „low cost reserves", etc. The choice of parameters to be used as measurements of the coordinate system is in compliance with the underlying logistics strategy of the organization, the improvement goal set forth, as well as with the necessity to follow up the deviations in their values.

In order to perform a correct comparison, it is necessary the target vector to be divided into partial target vectors, similar to the supply logistics process in the organization. It is assumed that the target vector visualizes an „ideal” supply logistics process running at competitions. Similar to the existing supply logistics process in the company it is also built up of certain number of sub-processes (partial target vectors). Their number and continuance are unknown. This information may be providing by the feedback or by other specialized sources (specialized literature, newspapers, magazines, scientific conferences, Internet sources, etc) (Sexton, 2011). On this basis three approaches to determining the partial target vectors can be distinguished. 
The first approach when there is information about the value of at least one coordinate of at least one partial vector is used. In the calculation of other vector coordinates it is assumed to be constant. From the coordinates of the target vector, constant value of the known partial vector coordinates is subtracted, so one of the partial target vectors is formed. The residue between the other partial target processes is divided equally, assuming that their number is equal to the number of actual partial vectors (sub-processes) of supply logistics.

If there is information about the coordinates of a target partial process applies the second approach. The number of target partial supply logistics processes and number of real sub-processes is assumed to equals again. The other partial target vectors are obtained by dividing equally the residue between the coordinates of the target vector and the known partial target vector.

In absence of specific data for the target sub-processes running unto competitors the third approach is applied. The number of target partial vectors and number of partial real vectors ( $n$ ) are assumed to equals again. Thus, each target sub-process $\left(\mathrm{SP}_{\mathrm{n}, \mathrm{T}}\right)$ can be calculated by division the coordinates of the target process $\left(P_{T}\right)$ and $1 / n$. „Averaged” partial target vectors, which are identical among themselves are created (Figure 1.). The coordinates of each averaged target supply logistics sub-process under the formula (formula 1) are derived.

$$
\begin{aligned}
\Delta_{a b s}=\frac{1}{n} P_{T}-S P_{i, j, R} & =\left(c_{1,1, T} ; c_{2,1, T} ; \ldots ; c_{m, n, T}-c_{1,1, R} ; c_{2,1, R} ; \ldots ; c_{m, n, R}\right)= \\
& =\left(d_{1,1} ; d_{2,1} ; \ldots ; d_{m, n}\right)
\end{aligned}
$$

where

$n$ - number of partial real and target vectors.

When the vector describing the absolute target of improvement of each sub-process has been calculated, it is required to determine the priority for improvement of those sub-processes. For this purpose it is necessary to introduce a coefficient that describes the necessity of supply logistics sub-process optimization in all measurements $-\delta_{j}$, which is calculated by formula 2 .

$$
\delta_{j}=\sum_{i=1}^{m} d_{i, j}
$$

where:

$d_{i, j}$ - measurementi to vectorj

$j=1 \ldots n-$ number of considered sub-process,

$i=1 \ldots \mathrm{m}$ - number of considered measurement. 


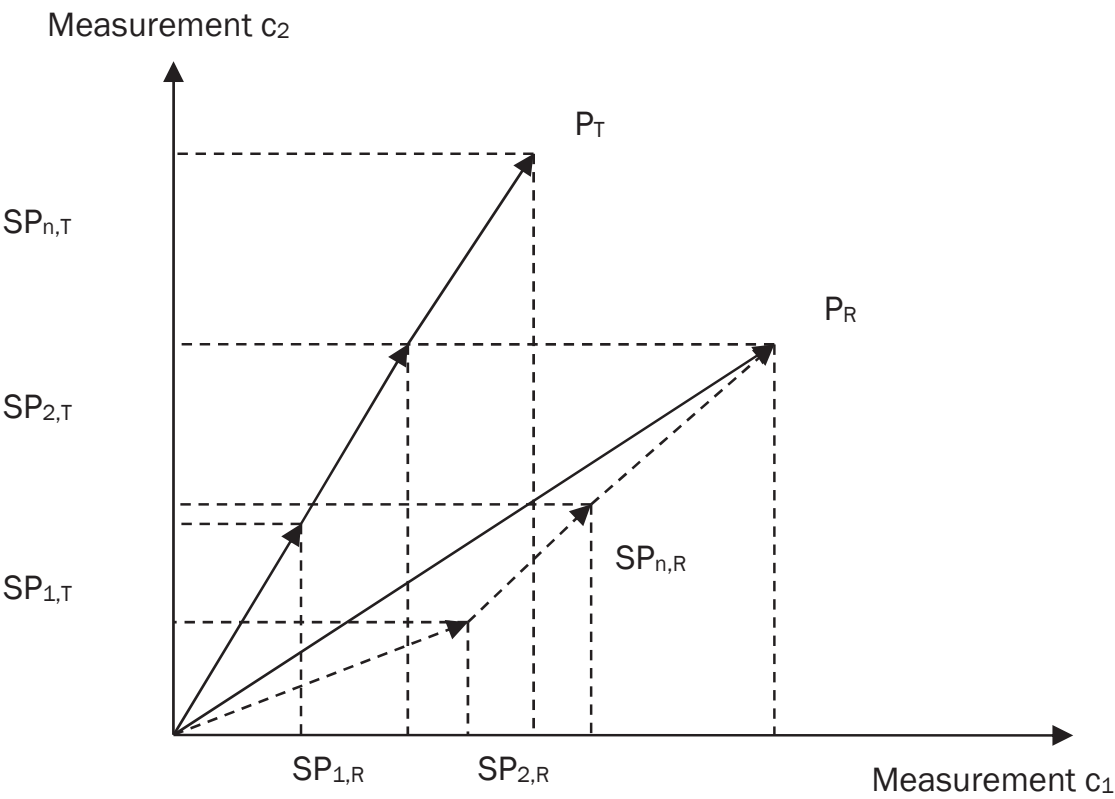

$\mathrm{P}_{\mathrm{T}}$ - target process; $\mathrm{P}_{\mathrm{R}}$ - real process; $\mathrm{SP}_{1, \mathrm{R}}, \mathrm{SP}_{2, \mathrm{R}}, \ldots \mathrm{SP}_{\mathrm{n}, \mathrm{R}}-$ real sub-process; $\mathrm{SP}_{1, \mathrm{~T}}, \mathrm{SP}_{2, \mathrm{~T}} \ldots \mathrm{SP}_{\mathrm{n}, \mathrm{T}}-$ target sub-process; $S P_{i, j}$ - coordinates $i$ to vector $j ; c_{i}$ - measurements of the processes; $j=1 \ldots n$ - number of considered sub-processes; $i=1$... $\mathrm{m}$ - number of considered measurements

Figure 1. Real and Target supply logistics process visualization

The methodology for calculation of the coefficients $\Delta_{a b s}$ and $\delta_{j}$ can be presented as an algorithm consisting of three blocks (Figure 2.). In block "A" a vector of the deviations of each supply logistics sub-process is calculated. In block "B" each coordinate of newly created vector $\left(\Delta_{a b s}\right)$ is compared to zero. This is the way to assessment the necessity of improvement of the real sub-processes. Further, it can be calculated with how many units the coordinates of the partial real vectors should be increased or reduced. In block "C" whether the measurements are trade-off is checked and depending on that $\delta_{j}$ representing the "absolute" target of sub-process improvement in all its measurements is calculated. In addition, the values of $\delta_{j}$ are ranked in descending order by size.

Step 1 - Determining the coordinates of all real and target partial vectors, which build the real process (vector) of the supply logistics in the organization and the target one at all studied (examined) measurements.

Step 2 - Calculation the difference between coordinates of the partial target and real vectors $-\Delta_{\text {abs }}$. 
Step 3 - Check of the nature of all measurements. If all characteristics of the supply logistics processes are maximizing ${ }^{4}$, algorithm continue to step 4 . Otherwise, all values of the new vector's coordinates $\left(\Delta_{a b s}\right)$ are multiplied by $(-1)$.

After those actions and calculation of the values of $d_{i, j}$, step 3 and block $A$ of the algorithm ends.

Step 4 - Comparison with zero the newly calculates vectors' coordinates, describing the „absolute” goal of improvement (Table 1). If the coordinate of the newly created vector is bigger than zero, then the target sub-process $s_{\mathrm{j}}$ in measurement $t_{i}$ is more efficient than the real one. In this case, optimization of the respective measurement of the real supply logistics sub-process is needed and algorithm continue whit step 5 in block $C$. In case that $d_{i, j}$ is less than zero, it means that the existing sub-process in $_{j}$ measurement $t_{i}$ is more efficient than the target one $e_{j}$ and improvement is not needed. In the third case $d_{i, j}=0$, which means that the real supply logistics sub-process ${ }_{j}$ is as efficient, as the target one ${ }_{j}$ in measurement $t_{\text {. }}$ Again optimization is not needed.

Table 1. Interpretation of " $\mathrm{d}_{\mathrm{i}, \mathrm{j}}$,

\begin{tabular}{|c|c|c|}
\hline correlations & interpretation & \\
\hline$d_{i, j}>0$ & $\mathrm{SP}_{\text {Target }}>\mathrm{SP}_{\text {Real }}$ & $\begin{array}{l}\text { The target sub-process in measurementi is more efficient than } \\
\text { the real one. }\end{array}$ \\
\hline$d_{i, j}=0$ & $\mathrm{SP}_{\text {Target }}=\mathrm{SP}_{\text {Real }}$ & $\begin{array}{l}\text { The real sub-process in measurement; is as efficient as the target } \\
\text { onej. There isn't necessity of improvement. }\end{array}$ \\
\hline $\mathrm{d}_{\mathrm{i}, j}<0$ & $\mathrm{SP}_{\text {Target }}<\mathrm{SP}_{\text {Real }}$ & $\begin{array}{l}\text { The real sub-process in measurement; is more efficient than } \\
\text { the target one. There isn't necessity of improvement. }\end{array}$ \\
\hline
\end{tabular}

Step 5 - Checking for trade-off about all of measurements. In this situation it is assumed that the deviations in the various measurements are balanced among them, since the characteristics of the supply logistics process (vector) contribute to different extent for the achievement of the supply logistics' goal. If all examines measurements are trade-off, the coefficient $\delta_{j}$ is calculated in step 6. Otherwise, proceed to step 7, and step 6 is not performed.

\footnotetext{
${ }^{4}$ Maximizing measurements are these, the values of which should be increased as a consequence of the improvement, and minimizing - those, the values of which should be reduced. Differentiating the vectors' parameters into "maximizing” (e.g. „accuracy of deliveries from suppliers”, „time of supply”, etc.) and „minimizing” (for instance „low process's costs”, „low cost reserves", etc.) is done on an earlier stage of the improvement. The differentiation in accordance with the strategic goals of the organization is done.
} 
Step 6 - Calculation the coefficient $\delta_{j}$ by formula 2 .

Step 7 - Checking for lack of offset between all of the characteristics of the supply logistics' process. If this is so, algorithm passes to step 10. It is assumed that if one of measurement needs to be improved, then the entire real process needs improvement. There is a third case in which, part of the measurements are trade-off, and part of them - aren't. Then, it is need to make subsequent verification of measurements which aren't trade-off in step 8.

Step 8 - Checking for existence of measurements which aren't trade-off and at the same time there are no need of improvement. If there are such measurements algorithm proceed with calculation of coefficient $\delta j$ only for the measurements that are trade-off in step 9. Otherwise, it pass to step 11. Step 9 - Calculation of coefficient $\delta_{j}$ only for measurements that are tradeoff and need optimization. The coefficient is marked with $\delta_{j}{ }_{j}$ and calculated by formula 3. For this purpose, the measurements of supply logistics' processes are divided into two sets - superset $C_{i}$ describing all measurements and subset $A_{q}$ describing only measurements that are trade-off. $A_{q}$ is subset of $C_{i}$ (all examined measurements) and its elements assume values for $q=1, \ldots, m$. The coefficient is $\delta_{j}^{\prime}$ is calculated like $\delta_{j}$, but only for those measurements that are trade-off. After calculating the coefficient algorithm passes to step 11.

$$
\delta_{j}^{\prime}=\sum_{q=1}^{m} d_{q, j}
$$

where

$d_{q, j}$ - measurementi to vector ${ }_{\mathrm{j}}$,

$j=1 \ldots n-$ number of considered sub-process,

$q=1 \ldots m-$ number of considered measurement,

Step 10 - The coefficient $\delta_{j}$ accepts the values of coordinates of vector $\Delta_{\text {abs }}$ which has need of improvement.

Step 11 - By the last operation in this block all values of $\delta_{j}$ are ranked by size in descending order. A sub-process of supply logistics which coefficient is highest gets rank "one”. It must be optimized first, since in practice is "outermost" from the set forth target process.

Then the algorithm stops.

$$
\Delta_{\mathrm{abs}}:=\frac{1}{n} P_{i, T}-S P_{i, j, R}:=\left(d_{1,1} ; d_{2,1} ; \ldots ; d_{m, n}\right)
$$




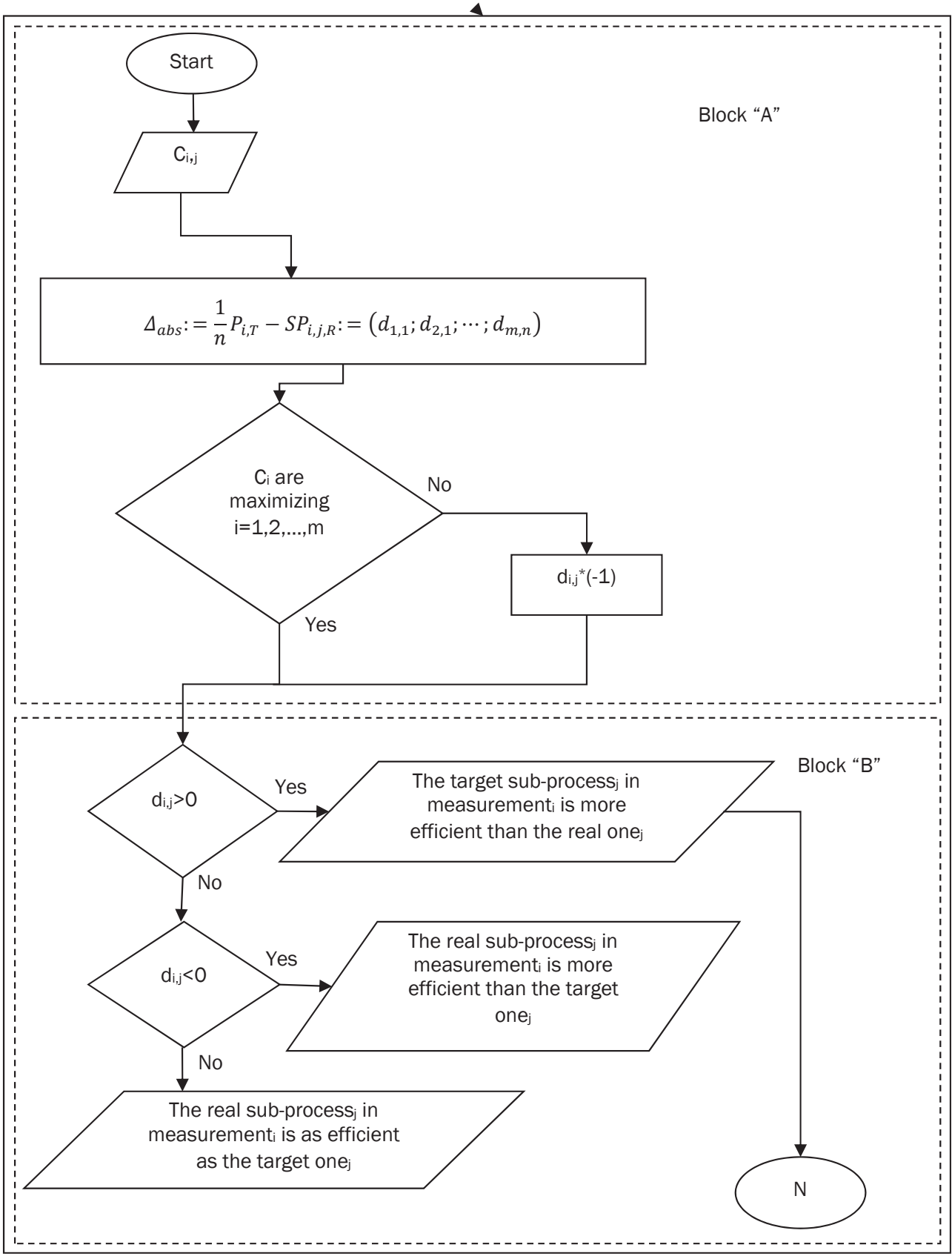




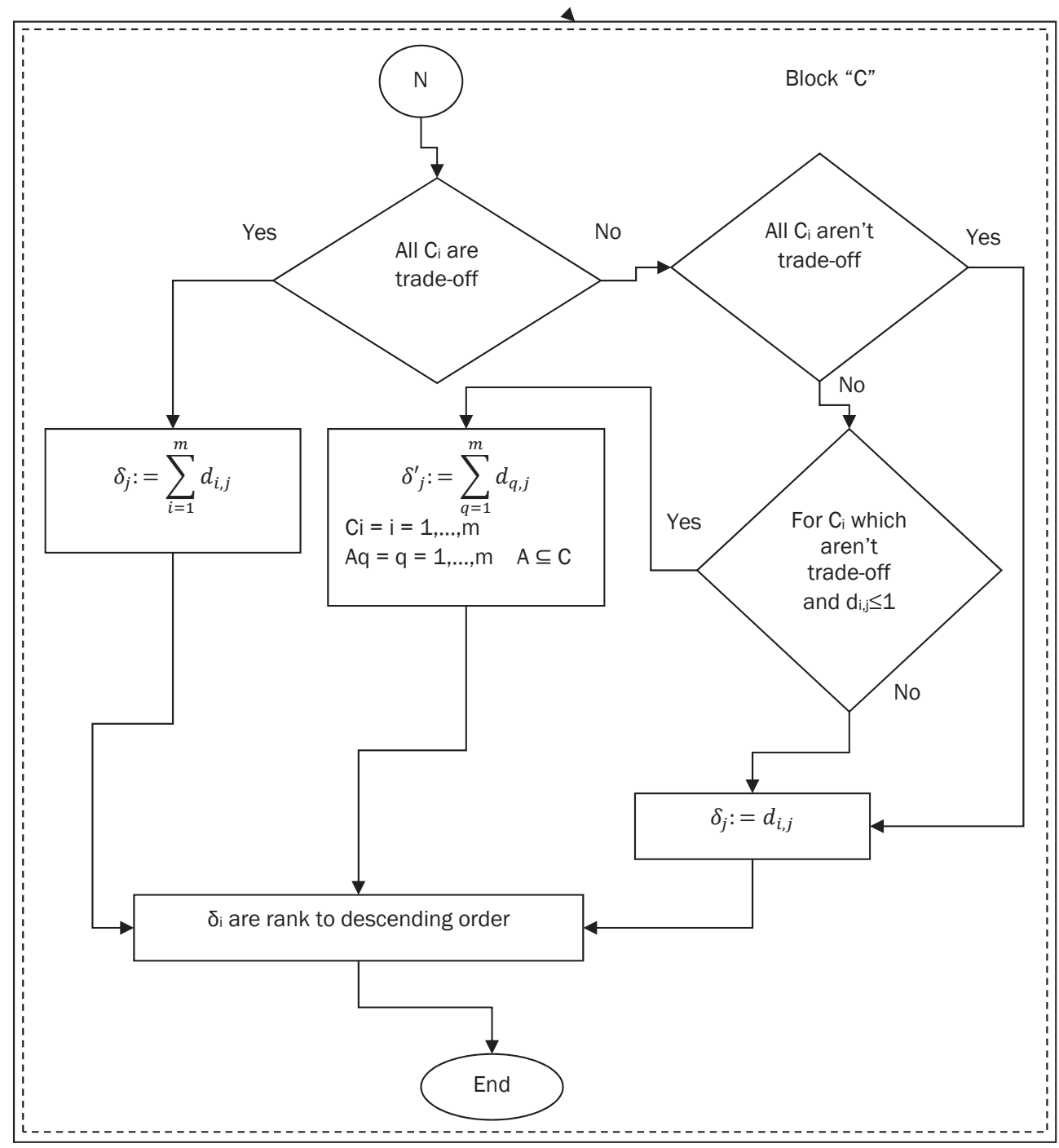

Figure 2. Algorithm for assessment of priority of supply logistics sub-process improvement

\section{Conclusion}

In the present paper an approach that can determine the priority of supply logistics sub-processes improvement in the organization was presented. It is based on establishing the efficiency of the real sub-processes compared to the set forth target efficiency. First it must be calculate a vector describ- 
ing the necessity of sub-process improvement in each measurement $\left(\Delta_{\text {abs }}\right)$. Depending on the obtained coordinate's values of this vector, a conclusion whether optimization of the real sub-processes is necessary is drawn. Subsequently, it can be determine the actual numeric value, by which to correct the coordinates of the real partial vectors under the relevant measurements. Second, coefficient representing the need for each supply logistics sub-process optimization in all its measurements $\left(\delta_{j}\right)$ is calculated. The resulting numerical values are arranged by size in descending order, and then must be put ranks to them. The sub-process with rank "one" must be optimized first.

The main advantage upon the application of this approach is that the measurements, under which the optimization is done, can be m-number as per the actual necessity. In order to determine $\Delta_{a b s}$ and $\delta_{j}$ only the subtraction and add operation is used, which simplifies the calculations. The algorithmic presentation of the entire methodology makes it possible to review and evaluate all possible combinations of the coordinate values of the vector describing the "absolute" necessity of supply logistics improvement. This way, integrity of the observation and representativeness of the defined conclusions is achieved. Main shortcoming of the described approach is that in case of lack of sufficient information, the target vectors are "averaged”. In some cases those "averaged target vectors" deviate substantially from the real partial target vectors. This could lead to "distortion" of the derived results at the end of the algorithm and to wrongful conclusions about the condition of the real supply logistics sub-processes.

The identification of the priority of sub-processes improvement represents the third stage of the supply logistics optimization process. The realization of all three stages of the supply logistics optimization process (assessment of overall necessity of improvement, assessment of the necessity and priority of the sub-process improvement) could lead to achievement of efficient and stable improvements of the supply logistics processes in the organization.

\section{References:}

Angelov, K. (2008). Business process reengineering (in Bulgarian), TU Sofia, Sofia.

Brüggemann, J., Heinrich, B., Sobczak, R. (1998). Mathematik, Cornelsen Verlag: Berlin.

Deckler, G. J. (2003). Achieving Process Profitability: Building the IT Profit Center, iUniverse Inc. . 
Dimitrov, Iv. (2004). Logistics Management (in Bulgarian), University „Prof. Dr. Assen Zlatarov" - Burgas, Burgas.

Haist, F. (2001). Qualität im Unternehmen: Prinzipien, Methoden, Techniken, Carl Hanser Verlag: München.

Harmon P. (2007). Business Process Change, Morgan Kaufmann Publishers. Harrington, H. (1991). Business Process Improvement, McGraw-Hill: New York. Lowenthal, J. N. (2003). Defining and Analyzing a Business Process: A Six Sigma Pocket Guide, ASQ Quality Press.

McDonald, M. (2010). Improving Business Process, Harvard Business School Publishing.

Ould, M. O. (2006). Business Process Management. A Rigorous Approach, Antony Rowe Ltd.: Chippenham.

Papula, L. (2001). Mathematik für Ingenieure und Naturwissenschaftler, Friedrich Vieweg und Sohn Verlagsgesellschaft: Braunschweig.

Portougal, V., Sundaram, D. (2006). Business Process. Operational Solutions for SAP Implementation, IRM Press.

Rosemann, M. (2006). Koplexitätsmanagement in Prozessmodellen, Gabler Verlag, Wiesbaden.

Sexton, D. (2011). Trump University. Branding 101: How to Build the Most Valuable Asset of Any Business, Locus. 